Por:

\title{
Elementos Predisponentes, a La Violencia Doméstica
}

Candidata Doctoral Irma Escudero Vaca. Profesora Titular de Salud Mental, Departamento de Salud Mental, Candidata Doctoral en Enfermería con Énfasis en Salud Internacional. Facultad de Enfermería, Universidad de Panamá. E mail: irmajev@yahoo.com

Dra. Lydia Gordón de Isaacs. Departamento de Salud de Adultos. Facultad de Enfermería. Universidad de Panamá. Email: isaacs@cwpanama.net

\section{Resumen}

Hace un tiempo atrás, la violencia doméstica era considerada como una conducta normal y aceptada, para muchas familias de diferentes culturas. Sin embargo, con el transcurrir del tiempo y gracias a muchas investigaciones, fueron identificados los prejuicios y daños físicos y emocionales que causa la violencia doméstica a las víctimas y a los miembros de la familia.

El hogar es el lugar donde se debe cuidar y proteger a sus miembros, pero en ocasiones es el lugar más violento y donde ocurren situaciones de violencia extrema, hasta llegar a la muerte. Son muchos los factores que en diferentes investigaciones se han encontrado relacionados a la violencia doméstica, Perluz, (2017); Fiestas, Rojas, Gushiken, Gozzer, (2012); Vaiz Bonifaz y Nakano, (2004); Alencar-Rodríguez y Cantera, (2013). En este artículo queremos abordar el consumo del alcohol, la infidelidad y la baja autoestima de las víctimas como factores que predisponen para que se presente la violencia doméstica. Este artículo surge de las entrevistas efectuadas a las tres primeras participantes en el estudio, que se realiza actualmente sobre el tema de violencia doméstica.

Palabras claves: Violencia doméstica, Alcoholismo, Infidelidad, Autoestima, Mujeres, Cultura.

\section{Predisposing Elements, to Domestic Violence}

\begin{abstract}
Some time ago domestic violence was considered as a normal conduct and accepted by many families of different cultures. However, many research has identified the prejudices and physical and emotional damage cause by domestic violence to the victims and to the members of the family. The home is one of the scenarios that more frequently presents Domestic Violence. In spite of the fact that the home that is the place where you must care for and protect your family members, in occasions is the most important place that is violated and where situations of extreme violence, occurs sometimes ending in death.

There are many factors that in different investigations have found related to domestic violence, Perluz, (2017); Fiestas, Rojas, Gushiken, Gozzer, (2012); Vaiz Bonifaz y Nakano, (2004); Alencar-Rodríguez y Cantera, (2013).

In this article we will deal with the consumption of alcohol, the infidelity and low self-esteem of the victims as factors that predispose to domestic violence. This article
\end{abstract}


emerges from interviews that were carried out the first three participants in the study that is conducted at this moment related to domestic violence.

Key words: Domestic Violence, Alcoholism, Infidelity, Self-esteem, Women, Culture.

\section{Elementos Predisponentes À Violência Doméstica}

\section{Resumo:}

Algum tempo atrás, a violência doméstica foi considerada um comportamento normal e aceito para muitas famílias de diferentes culturas. No entanto, com a passagem do tempo, e graças a muitas investigações, os danos físicos e emocionais e os danos causados pela violência doméstica às vítimas e aos membros da família foram identificados.

O lar é o lugar onde o cuidado deve ser tomado e seus membros protegidos, mas as vezes é o lugar mais violento e ocorrem situações de extrema violência, até a morte. Existem muitos fatores que em diferentes investigações têm sido relacionados à violência doméstica (Perluz, 2017, Fiestas, Rojas, Gushiken, Gozzer, 2012, Vaiz Bonifaz e Nakano, 2004, Alencar-Rodríguez e Cantera, 2013).

Neste artigo queremos abordar o consumo de álcool, infidelidade e baixa autoestima das vítimas como fatores predisponentes para a violência doméstica. Este artigo decorre das entrevistas realizadas com os três primeiros participantes no estudo que atualmente é realizado sobre o tema da violência doméstica.

Palavras Chaves: Violência Doméstica, Alcoolismo, Infidelidade, Mulheres, Cultura.

\section{Introducción}

La información recabada en este artículo surge de la investigación doctoral que se realiza en la actualidad y que tiene como objetivo central, conocer las creencias y prácticas de salud de las mujeres hispano-indígenas víctimas de violencia doméstica de la ciudad de Santiago de Veraguas, capital de la provincia de Veraguas, la más grande de las diez provincias de Panamá.

La Provincia de Veraguas, su ciudad es semi-urbana, situada a orillas de la carretera panamericana, con una población aproximada de 222,641 habitantes (Dirección de Estadística y Censo, 2010).

La provincia de Veraguas limita con la Comarca Ngäbe Buglé, una de las cinco comarcas indígenas del país. La población veragüense está compuesta en su gran mayoría por mestizos (hispano indígena). Este dato del contexto donde se realizó la investigación es importante ya que el componente cultural influye en las expresiones de las personas.

La violencia doméstica es un fenómeno que ocurre en todos los países y nuestro país no escapa de ello. Se han hecho muchos estudios, que generalmente han integrado 
información de los dos actores, víctima y agresor. En este documento, abordamos este problema desde la perspectiva de las mujeres hispano-indígenas víctimas de violencia doméstica. En esta información pudimos identificar tres factores comunes a todas, referenciados por su relación con la violencia doméstica: la baja autoestima de la víctima, la infidelidad del agresor y el consumo de alcohol.

Este estudio responde al paradigma cualitativo; el método utilizado es la etnoenfermería, método para investigar el cuidado de enfermería, según Leininger (1991) citado por Gordón de Isaacs (2015):

"La ethnoenfermería fue el primer método de investigación propuesto para la disciplina de Enfermería, el mismo se centra en las personas. Este método busca estudiar las percepciones que tienen las personas acerca de las características del cuidado humano, desde sus creencias y valores culturales". (p.28).

La utilización de la etnoenfermería sirve para desarrollar conocimiento propio para la disciplina de enfermería al descubrir las prácticas de cuidado que realizan las mujeres hispano-indígenas víctimas de violencia doméstica. Para la recolección productiva de los datos se utilizó la entrevista a profundidad, observaciones, notas de campo y las reflexiones acerca de la información recabada. Se estableció contacto con las participantes clave entrevistadas, en un Centro de Salud en la ciudad de Santiago de Veraguas, con el apoyo de la trabajadora social del centro, que atiende a mujeres víctimas de violencia doméstica. Se firmaron los consentimientos informados para realizar las entrevistas y se trabajó en condiciones de privacidad.

\section{Víctimas de violencia doméstica}

La familia desde mucho tiempo atrás, se consideró un espacio privado; nadie podía opinar ni intervenir en su dinámica. acerca de los tratos dados a los hijos y a las mujeres. Esto nos lo confirma Lorente y Lorente, (1998), citado por Patró Hernández y Limaña Gras (2005), cuando nos dice que: "Las creencias y mitos culturales asociados al sistema patriarcal han legitimado desde tiempos remotos el poder y la dominación del marido hacia la mujer y los hijos, despojando a estos de todo derecho legal, económico o social" (p.11). La esposa y los hijos fueron víctimas de golpizas, insultos, ya que eran una propiedad del marido, él podía hacer lo que quisiera para mantener el control sobre ellos.

Así que cuando hablamos de las víctimas nos referimos a las mujeres, así como nos lo señala Blitchtein Wernicke y Reyes Solar. (2012) cuando dicen que:

"Estudios como los realizados por Hindi en diez países

(6) y por García-Moreno y su equipo en 2006, en quince sitios ubicados en diez países, dos de ellos en Perú, coinciden en la identificación de una elevada prevalencia de violencia física o sexual de pareja. Las victimas más 
frecuentes de violencia doméstica son los grupos más vulnerables, mujeres, niños, niñas y ancianos. Muchas investigaciones señalan que las mujeres son el grupo más victimizado hacia la mujer" (p. 35).

Las mujeres son identificadas como los grupos más vulnerables para sufrir de violencia doméstica en el mundo según el Informe de la Organización Mundial de la Salud (OMS, 2013), el cual dice que: "En todo el mundo, casi un tercio (el 30\%) de todas las mujeres que han mantenido una relación de pareja han sido víctimas de violencia física y/o sexual por parte de su pareja. En algunas regiones, esta cifra puede llegar a ser de hasta el $38 \%$ " (p.1). Además, en algún momento de la vida de la mujer se le presenta un incidente de violencia doméstica, apunta también la OMS (2013):

"Una de cada 3 mujeres sufre violencia física o sexual infligida por la pareja en algún momento de su vida. Estudios realizados en diferentes países demuestran que el porcentaje de mujeres de 15 a 49 años que han sufrido violencia física o sexual por parte de su pareja a lo largo de su vida se sitúa entre el 15\% y el 71\%" (p.2).

La mujer sigue estando en un nivel de subordinación al hombre; son esquemas que comparten hombres y mujeres y que resultan de procesos de socialización y de aprendizaje, según los cuales las mujeres son propiedad de esposos, maridos, novios o padres. Esto hace que los hombres se sientan dueños de ellas, de sus pensamientos, sentimientos y comportamientos y que consideren que las mujeres están para satisfacer las necesidades de los hombres. La violencia es una conducta que a pesar de los años se les sigue enseñando y modelando a los niños y niñas como nos señala Rodríguez, H. (2008) cuando afirma que a violencia es una manifestación cultural aprendida, y que en el seno familiar se sustentan poderes que la legitiman, desde entender al otro u otra como propiedad y no como persona con libertad de decisión.

Por otro lado, tanto las mujeres como los hombres enseñan y modelan a sus hijos e hijas comportamientos de subordinación de las mujeres a los hombres y esto ocurre aun cuando las propias mujeres se ocupen mayoritariamente de la crianza de los niños. Es que se trata de una especie de mandato social, de un ciclo que se repite en muchas sociedades permitiendo que estas normas se arraiguen más en el comportamiento social aceptado por la sociedad.

Es el arraigo cultural que tiene la conducta violenta, lo que hace que se sigua aceptando dicho comportamiento como normal y que la violencia de género se perpetúe pese a os grandes avances tecnológicos y sociales de hoy.

\section{El Estudio}

Para conocer las creencias y las prácticas culturales de salud de las mujeres víctimas de violencia doméstica se utilizó la entrevista en profundidad, para recoger la perspectiva de las mujeres acerca de las prácticas culturales de salud que ellas utilizan para cuidar su salud física y mental y así evitar los daños, que la violencia vivida, trae consigo. 
Este artículo se refiere a las tres primeras entrevistas a mujeres víctimas de violencia doméstica, que viven en la ciudad de Santiago. Los criterios para escoger a las participantes del estudio fueron: que hubieran vivido alguna situación de violencia doméstica, que residieran en la ciudad de Santiago, de la provincia de Veraguas, de la República de Panamá, y que consintiera de manera informada en hablar de la situación de violencia doméstica vivida.

Las edades de las participantes estaban entre 28 y 35 años; dos estaban casadas por lo civil y una unida; dos poseen estudios universitarios no completados y otra formación secundaria completa. Las tres mujeres generaban ingresos económicos al hogar, ya que trabajaban; no dependían de sus conyugues para el sustento.

Dos de las informantes se desempeñaban como docentes y una era vendedora a domicilio. En cuanto al cuadro de violencia vivido, las tres habían sufrido violencia física, una con empujones y violencia verbal y psicológica; la segunda con golpes físicos, violencia verbal y psicológica; y la tercera había sido agredida con arma blanca en el abdomen, además de violencia verbal y psicológica. Las tres mujeres habían tenido varios episodios de violencia doméstica y se habían separado y vuelto a reconciliar con el agresor, en varias ocasiones.

El método de etnoenfermería señala que es necesario realizar el análisis preliminar después de cada entrevista para lograr la saturación, es decir para saber cuándo hemos completado la colección de datos. Al concluir la tercera entrevista seguían saliendo datos nuevos, por lo que siguió hasta alcanzar siete (7) entrevistas. Este estudio, se refiere a los datos preliminares que arrojaron las tres primeras entrevistas.

Las tres participantes del estudio identificaron el consumo de alcohol del agresor como un factor detonante para la violencia doméstica. Una de las participantes manifestó que su pareja se tornaba mucho más violento con el consumo de alcohol. Por otro lado, una participante manifestó que ella salía con sus amigas y que consumía alcohol en forma ocasional, para liberarse del estrés.

En el momento de la entrevista las tres mujeres estaban separadas de sus cónyuges. Las tres refieren tener conocimiento de la infidelidad de los mismos, además de manifestar que los celos y la infidelidad eran uno de los motivos por los cuales discutían y se presentaba la violencia.

Otro elemento importante es la baja autoestima por parte de las víctimas. Una de las participantes sentía que su vida giraba alrededor de su pareja; ella era menos; él era un hombre estudiado y ella no había terminado su carrera. Las otras dos participantes manifestaron que sus parejas las hacían sentirse menos, que no eran atractivas, ni sabían hacer bien las cosas.

En resumen, tres factores son comunes a las experiencias de violencia doméstica vivida por nuestras informantes clave: la baja auto estima por parte de la víctima, la infidelidad masculina y el consumo de alcohol por parte del agresor.

\section{Consumo de Alcohol por Parte del Agresor}


El consumo de alcohol y drogas es uno de los factores predisponentes de la violencia doméstica, tal como han identificado muchas investigaciones sobre el tema, de manera que entre más se consume alcohol por parte del agresor más riesgo hay de sufrir de violencia. Así lo señalan Blitchtein Reyes (2012): "las mujeres con parejas que toman alcohol hasta la embriaguez frecuentemente, tienen siete veces más riesgo de sufrir violencia física por su pareja, que las que no lo tienen" (p.40). Incluso las mismas víctimas identifican el alcohol como un detonante para la violencia (Bott, Guedes, Goodwin y Adams, 2012). Estos autores afirman que: "las mujeres señalan muchas situaciones diferentes que 'desencadenan' violencia de parte del compañero íntimo, pero en casi todos los entornos el consumo de alcohol por parte de este desempeña un papel importante" (p.18).

Así lo pusieron de manifiesto las participantes cuando dijeron:

"él siempre toma demasiado y esto siempre... altera a las personas obviamente de la parte... como le puedo explicar la parte lúcida a la parte que no es lúcida. Se torna agresivo, él ya estaba tomando bastante...Forcejeamos, mucho, "el me apretó tanto el cuello que yo sentía que se me escapaba la vida” C1

"Él llegaba tomado de la calle, tarde en la noche, discutíamos por cualquier cosa y comenzaba la violencia, el alcohol lo ponía más agresivo, uno pierde ese sentido de ver la vida bonita, de luchar, de echar para adelante, de que existe un futuro". $\mathbf{C 2}$

"El salía con otras mujeres, así comenzó la violencia de mi esposo hacia mí, me gritaba, me insultaba, me pegaba cuando venía a la casa borracho, así que puse una denuncia”.C3

Aunque las tres participantes manifiestan que, en las situaciones de violencia vivida, el agresor estaba bajo la influencia del alcohol, teniendo más probabilidades de sufrir violencia doméstica, cabe señalar que no se puede justificar la violencia por el consumo del alcohol.

El consumo de alcohol genera muchos problemas físicos y mentales para el consumidor y para su familia. El alcoholismo está asociado a comportamientos violentos, hay muchos estudios que identifican el alcoholismo como un factor de riesgo para la violencia doméstica, así nos lo indica Herrera Paredes y Arena Ventura (2010) cuando nos dice que:

“...el alcoholismo registra un gran porcentaje de situaciones en que las mujeres son agredidas por sus compañeros conyugales, que están bajo el efecto del alcohol. De esta forma, se verifica una mayor vulnerabilidad femenina frente a la violencia familiar y también que la presencia del alcohol en el hogar se relaciona de manera importante con la agresión familiar, principalmente contra las mujeres, siendo una variable de riesgo" (p. 560), 


\section{Infidelidad y Su Relación con la Violencia Doméstica}

Cuando hablamos de la infidelidad casi siempre nos referimos a las relaciones maritales o de noviazgo, a pesar de que el término se puede aplicar a la falta a los valores y principios éticos personales. En esta ocasión estamos tratando específicamente de la infidelidad conyugal, situación que involucra muchos aspectos, emociones y sentimientos que pueden estimular comportamientos violentos. Se define infidelidad conyugal según Hernández y Pérez (2007) como: "una construcción sociocultural resultado de la instauración del patriarcado al derogar el derecho materno, y por tanto comporta en sí mismo, significados claramente diferenciados para hombres y mujeres" (p.3).

Por otro lado, según Buss et al (1999) citados por Canto Ortiz, García Leiva, Gómez Jacinto (2009) hay diferencias emocionales según el sexo, frente a la infidelidad: "los hombres se sienten más preocupados que las mujeres por una hipotética infidelidad sexual de sus parejas ... las mujeres se sienten peor que los hombres ante una hipotética infidelidad emocional" (p.40). Esto explica, en parte, que muchas de las situaciones de violencia doméstica se presenten por una supuesta infidelidad de la mujer; la preocupación de los hombres, sus inseguridades, se reflejan en comportamientos violentos que la mayoría de las veces desencadenan en un hecho de violencia doméstica.

Un aspecto que no podemos perder de vista es el que nos plantean Agoff, Rajsbaum, Herrera (2006) acerca de los intereses que están detrás de una unión familiar y que la infidelidad desequilibra:

“... la infidelidad no debe situarse necesariamente en el ámbito de los sentimientos de ambos cónyuges, sino en el de los intereses que la unión conyugal representa para cada uno: se trata de que ella no transgreda una norma que lo colocaría a él en una situación de poca hombría y de dignidad manchada. En el caso de la mujer, la amenaza de que él le sea infiel conlleva el riesgo de perder eventualmente la manutención para ella y sus hijos y/o el estatus social que le confiere tener una pareja estable y unida (p.311).

La infidelidad es un comportamiento que se agrava mucho más, ya que dificulta la convivencia y la búsqueda de soluciones pacíficas a los problemas del diario vivir que enfrentan las mujeres: según cuando nos dice que:

"La mayor parte de las mujeres conviven con el problema de la infidelidad masculina y de la violencia doméstica, que resulta agravado por las circunstancias que dificultan la sobrevivencia. La mayoría de las mujeres se somete y adopta un comportamiento pasivo, o expresa sus quejas generando nuevas discusiones y agresiones en la pareja, lo cual no contribuye a modificar el proceder del hombre, ni a preservar la integridad física y síquica de la mujer. Esto revela la dominación masculina ejercida en las relaciones entre ambos sexos, y caracteriza las asimetrías de las relaciones de género presentes en el 
matrimonio, reflejando las condiciones de sumisión a las que están expuestas estas mujeres" Cizino Da Trindade, De Almeida, Alves Rosendo, (2008): p. 1).

Los relatos de las participantes de nuestro estudio, identifican a la infidelidad asociada al maltrato por parte de su pareja, quien supuestamente la maltrata impulsado por cuestiones que van más allá de él:

“... me encuentro con un mensaje que él le había mandado a una mujer, y confirmaba lo que yo presentía que él andaba con otra mujer y como el acababa de llegar de un Congreso... yo me disgusto y yo estrello el celular en el suelo... luego él me agarra se lanza contra mí, trata de estrangularme" $\mathbf{C 1}$

"Él era indispensable en mi vida" yo le perdone las infidelidades, tanto que hay un hijo por fuera del matrimonio, yo obvie eso, cuando me di cuenta de eso, el amor de pareja se iba perdiendo y cuando él quiso retomar eso, cuando vio que yo no quería nada con él, yo a él lo respete tanto, yo nunca me metí con ningún otro hombre”.C2

"El comienza una relación con la hermana de una comadre mía, la mujer fue a mi casa, a decirme que tenía una relación de un año con mi esposo. Yo le reclame y él me "zarandeo" del brazo, me insulta, niega la relación”.C3

\section{Baja Autoestima de la Víctima de Violencia Doméstica}

La autoestima es la forma en que cada persona se valora a sí misma, como se siente consigo misma, con respecto a sus actuaciones, sus habilidades, sus potencialidades y como se siente valorada por las demás personas. Las mujeres partimos de una valoración inferior porque nuestra sociedad valora más al hombre que a la mujer, lo que incide en la autoestima. Se aprende en los hogares con roles tradicionales donde observan malos tratos a los miembros de género femenino.

Todos estos elementos condicionan nuestra autoestima. Por ello, cuando convivimos con un hombre agresor, nuestra autoestima baja, incluso hasta llegar a perder la propia identidad (me comporto como me indican, no sé qué hacer, ¿qué quiere que haga?). La autoimagen y el auto concepto forman parte de la autoestima, la confianza es importante para sentirnos bien, como nos dice Carneiro Vianna, Teodoro Bomfim y Chicone (2006):

"La satisfacción de necesidades de autoestima lleva al individuo a sentir confianza en sí mismo (en su valor, fuerza, capacidad y adecuación), que es más útil y necesario al mundo. La falta de satisfacción produce en el individuo un sentimiento de inferioridad, flaqueza e impotencia” (p.2). 
Una baja autoestima afecta la sensación de seguridad, una necesidad básica de toda persona que incluye el sentirse bien con ella misma y con su entorno, pues como indican: Carneiro Vianna, et al (2006):

"Las necesidades básicas están entrecruzadas y el bienestar depende de la seguridad: cuanto más seguros nos sentimos en el ambiente en que estamos circunscritos, nuestra autoestima estará siendo alimentada por la confianza y respeto, y consecuentemente se mantendrá elevada" (p.2).

En sus relatos las mujeres entrevistadas, asocian la baja autoestima con el maltrato por parte de la pareja, quien supuestamente las maltrataban porque ellas no hacían bien las cosas:

“...porque siempre yo me consideraba como inferior... Y en esta situación yo le creía lo él me decía por esto he vivido todo esto... él, era mi vida, pero me hacía sentir que, sin él, yo no podía vivir, no podía sobrevivir, y eso a lo que yo me aferraba a pensar” E1

"Mi esposo no quería que estudiara porque él no quería que yo tuviera aspiraciones, así que me salí de la universidad, porque él se frustraba y discutíamos mucho, no quería que él se molestara no quería más problemas". E2

"No me sentía bien como mujer, me sentía que no servía. Cuando yo le preguntaba, se tornaba violento, "tú no eres nadie para preguntarme nada", me empuja, me insultaba, me denigraba como mujer. Me sentía que no servía para nada, por eso me golpea, no soy una mujer”. E3

Se observa en el discurso de las víctimas que la falta de autoconfianza las vuelve dependientes y simultáneamente endiosan a la persona de la cual dependen. Esta situación hace que ellas no puedan identificar que el irrespeto e incluso las agresiones recibidas de esa persona admirada y necesitada, son formas de agresión que bajo ningún concepto ellas se merecen.

\section{Consideraciones Finales}

Mucha gente piensa que el problema de la violencia doméstica en el mundo no tiene solución; que está arraigado en las normas y conductas sociales y que no se puede cambiar. Pero cada persona es parte de la solución, en la medida en que rechacemos la violencia y favorezcamos los ambientes de paz y armonía, con medios no violentos para resolver dificultades.

Desde el enfoque cultural, la violencia doméstica se acepta como algo normal, actitud que constituye una conducta aprendida, pues la han visto en sus hogares, han crecido con esos comportamientos. La mujer maltratada se encuentra atrapada entre el agresor que abusa de ella y ese mismo agresor que suple sus necesidades y las de sus hijos. 
Son muchos los factores asociados a la violencia doméstica. De acuerdo con nuestro estudio, los hallazgos indican la baja autoestima de las víctimas, como consecuencia de los años de maltrato vivido, desde la infancia hasta el periodo adulto. Otro factor es el consumo de alcohol por parte del agresor, que al consumir alcohol, es un medio, para justificar su comportamiento violento escudando su cobardía con el consumo de alcohol, su impotencia para resolver problemas del diario vivir y como excusa para rehuir sus responsabilidades de hombre cabeza de hogar, posición que él como hombre solicitó y juro cumplir su palabra, ante una mujer que el escogió para ser la madre de sus hijos, lo que implica inconsistencia en su papel de hombre cabeza de familia con la responsabilidad adquirida de ser el proveedor de las necesidades de su familias, preservar la igualdad de derechos de todos los miembros de su familia y ser el guardián de la paz en el hogar.

Por último, la infidelidad del agresor o los celos por parte de ambos miembros de la pareja, resultan ser detonantes de la violencia en el hogar, que en ocasiones termina con la muerte de un miembro de la pareja. La violencia doméstica es un problema de todos y por ello todos los sectores de la sociedad deben participar en la planificación de programas de prevención de este problema, el sector educación, salud, administración de la justicia, medios de comunicación y servicios sociales.

\section{Referencias}

Agoff, C. Rajsbaum, A. Herrera, C. (2006). Perspectivas de las Mujeres Maltratadas Sobre la Violencia de Pareja en México. Revista de Salud pública. México. Recuperado de:

http://www.scielo.org.mx/scielo.php?script=sci_arttext\&pid=S0036-36342006000800011

Alencar- Rodríguez, R. Cantera, L. (2013). Del Laberinto Hasta el Camino Hacia la Recuperación de la Violencia. Revista Redalyc. Interamerican Journal of Psychology, vol. 47, num. 1, pp. 61-69. Sociedad Interamericana de Psicología. Austin, Organismo Internacional. Recuperado de:

http://www.redalyc.org/articulo.oa?id=28426980008

Blitchtein Winicki, Reyes Solar. (2012). Factores Asociados a Violencia Física Reciente de Pareja Hacia la Mujer en Perú, 2004-2007. Revista Perú Med Exp Salud Pública. Pag.35-43. Recuperado de:

http://www.ins.gob.pe/insvirtual/images/artrevista/pdf/rpmesp2012.v29.n1.a06.pdf

Bott, S. Guede, A. Goodwin, M. Adams, J. (2012). Violencia Contra la mujer en América Latina y el Caribe: Análisis comparativo de datos poblacionales de 12 países. Washington, D.C. OPS. Recuperado de: https://goo.gl/sBPwnJ

Canto Ortiz, J. García Leiva, P. Gómez Jacinto, J. (2009). Celos y Emociones: Factores de la Relación de Pareja en la Reacción ante la Infidelidad. Revista Redalyc Org. Athenea Digital, Universidad Autónoma de Barcelona España. Recuperado de: http://www.redalyc.org/articulo.oa?id=53712929003

Carneiro Vianna, L. Teodoro Bomfim, G. Chicone, G. (2006). Autoestima de las Mujeres que Sufrieron Violencias. Artículo Original. Revista Latino-am Enfermagem. Septiembre-octubre. Recuperado de: http://www.scielo.br/pdf/rlae/v14n5/es_v14n5a09.pdf

Cizino Da Trindade, R. De Almeida, A. Alves Rosendo, C. (2008). Infidelidad Masculina y Violencia Domestica: La Experiencia de un Grupo de Mujeres. Revista Ciencia y Enfermería XV. Pag. 36-46. Recuperado de: http://www.scielo.cl/pdf/cienf/v14n2/art06.pdf

Fiestas, F. Rojas, R. Gushiken, A. Gozzer, E. (2012). ¿Quién es la Víctima y Quién el Agresor en la Violencia Física entre Parejas? Estudio Epidemiológico en Siete Ciudades del Perú. Revista Peruana Medica 
Enfoque, Revista Científica de Enfermería, VOL. XXI, N 17 Julio a Diciembre 2017 ISSN 1816-2398 Impreso

Experimental y Salud Pública. vol.29 no.1 Lima. Recuperado de: http://www.scielo.org.pe/scielo.php?pid=S1726-46342012000100007\&script=sci_arttext

Gobierno Nacional de Panamá. (2013). Informe Panamá. Primera Reunión de la Conferencia Regional. Sobre Población y Desarrollo de América Latina. Montevideo, Uruguay del 12 al 15 de agosto Recuperado de: http://www.cepal.org/celade/noticias/paginas/9/50639/CRPD_Panama.pdf

Gordón de Isaacs, L. (2015). Cuidado Cultural: Investigación y Práctica. Panamá. Imprenta Universitaria. Universidad de Panamá.

Herrera Paredes, J.M. y Arena Ventura, C.A. (2010). Consumo de Alcohol y Violencia Doméstica Contra las Mujeres: un Estudio con Estudiantes Universitarias de México. Revista Latino-Am. Enfermagem. MayJun; 18(Spec):557-64 Recuperado de: http://www.redalyc.org/articulo.oa?id=281421939011

Hernández García, Y. Pérez Gallo, V. (2007). Un Análisis Feminista de la Infidelidad Conyugal. Revista Redalyc Org. pp. 113-119, Universidad Complutense de Madrid España. Recuperado de: http://www.redalyc.org/pdf/181/18153299008.pdf

Ortega-Ceballos, P. Mudgal, J. Flores, Y. Rivera-Rivera, L. Díaz-Montiel, J. Salmerón, J. (2007). Determinantes de Violencia de Pareja en Trabajadoras IMSS Morelos. Salud Pública de México. 2007;49(5):357-366. Recuperado de: http://www.scielo.org.mx/scielo.php?script=sci_arttext\&pid=S0036-36342007000500006

Patró Hernández, R. Limaña Gras, R. (2005). Víctimas de Violencia Familiar: Consecuencias Psicológicas en Hijos de Mujeres Maltratadas. Universidad de Murcia. Revistas Anales de Psicología. Vol. 21, $\mathrm{n}^{\mathrm{o}} 1$ (junio), 11-17. España. Recuperado de: https://search.proquest.com/openview/dd4a90e809c87feda7323612098540ba/1?pqorigsite $=$ gscholar $\& \mathrm{cbl}=1606360$

Perluz, S. (2017). La Violencia Familiar (conyugal/pareja) en las Ciudades de Cartagena y Barranquilla en el Caribe Colombiano. Pensamiento Americano Vol. 10 - No. 18 • Enero-Junio $•$ Corporación Universitaria Americana $\bullet$ Barranquilla, Colombia • ISSN: 2027-2448 • pp. 161-178, Recuperado de: http://www.coruniamericana.edu.co/publicaciones/ojs/index.php/pensamientoamericano/article/view/391

Rodríguez, Hortensia N. (2008). Violencia de Género en Cartagena de Indias: Crónicas e Imágenes de un Amor que se Revela. Tratos Inhumanos y Crueles. Revista Palabras. Recuperado de: http://revistas.unicartagena.edu.co/index.php/palobra/article/view/208/0

Vaiz Bonifaz, R. y Nakano, A. (2004). La Violencia Intrafamiliar, el Uso de Drogas en la Pareja, desde la Perspectiva de la Mujer Maltratada. Revista Latino Americana de Enfermagem Vol.12:433-438 Recuperado de: http://www.scielo.br/pdf/rlae/v12nspe/v12nspea20.pdf 